\title{
Energy-Efficient Short Packet Communications for Uplink NOMA-based Massive MTC Networks
}

\author{
Shujun Han, Student Member, IEEE, Xiaodong Xu, Senior Member, IEEE, Zilong Liu, Member, IEEE, \\ Pei Xiao, Senior Member, IEEE, Klaus Moessner, Senior Member, IEEE, Xiaofeng Tao, Senior Member, IEEE, \\ Ping Zhang, Fellow, IEEE
}

\begin{abstract}
The 5th generation (5G) mobile networks and beyond need to support massive machine-type communications (MTC) devices with limited available radio resources. In this paper, we study the power-domain non-orthogonal multiple access (NOMA) technology to support energy-efficient massive MTC networks, where MTC devices exchange information using sporadic and low-rate short packets. We investigate the subchannel allocation and power control policy to maximize the achievable effective energy efficiency (EE) for uplink NOMA-based massive MTC networks, taking into account of short-packet communication characteristics. We model the subchannel allocation problem as a multi-agent Markov decision process and propose an efficient Q-learning algorithm to solve it. Furthermore, we obtain the optimal transmission power policy by approximating the achievable effective rate of uplink NOMA-based short packet communications. Compared with the existing OFDMA scheme, simulations validate that the proposed scheme can improve the achievable effective EE of massive MTC networks with $5.93 \%$.
\end{abstract}

Index Terms-massive MTC, NOMA, short packet communication, energy efficiency, joint subchannel and power allocation

\section{INTRODUCTION}

Towards the 5th generation $(5 \mathrm{G})$ mobile networks and beyond, massive machine-type communications (MTC), aiming to provide seamless and ubiquitous wireless connections anywhere and anytime, have received tremendous research attention in recent years. According to the forecast of Cisco, by 2022, there will be 3.9 billion of MTC devices connected in a variety of vertical industries such as industrial automation, intelligent transportation, smart environment, smart city, smart

Copyright (c) 2015 IEEE. Personal use of this material is permitted. However, permission to use this material for any other purposes must be obtained from the IEEE by sending a request to pubs-permissions@ieee.org.

The work presented in this paper is also partly funded by the National Science and Technology Major Project No. 2018ZX03001029-004, the National Natural Science Foundation of China No. 61871045, the European Unions Horizon2020 research and innovation programme (Clear5G) under grant agreement No. 761745, and the Engineering and Physical Sciences Research Council (EPSRC) No. EP/P03456X/1. This works is also supported by BUPT Excellent Ph.D. Students Foundation CX2019308 and the China Scholarship Council. (Corresponding author: Xiaodong $\mathrm{Xu}$ )

Shujun Han, Xiaodong Xu, Xiaofeng Tao are with the National Engineering Laboratory for Mobile Network Technologies, Beijing University of Posts and Telecommunications, Beijing 100876, China (E-mail: hanshujun, xuxiaodong, taoxf@bupt.edu.cn )

Zilong Liu, Pei Xiao and Klaus Moessner are with Institute of Communication Systems, Home of 5G Innovation Centre, University of Surrey, Guildford GU2 7XH,UK (E-mail: zilong.liu, p.xiao, k.moessner@surrey.ac.uk )

Ping Zhang is with the State Key Laboratory of Networking and Switching Technology, Beijing University of Posts and Telecommunications, Beijing 100876, China (E-mail: pzhang @bupt.edu.cn ) health, smart home, security and public safety [1]. Although cellular networks provide several advantages for human-type communications (HTC), it is becoming increasingly challenging for them to support massive MTC devices with limited radio resources.

Sporadic and low-rate short packet communication is a major feature of massive MTC networks. Typical applications of short packet communication in massive MTC networks are data sensing and collection, remote control and maintenance. Basically, the data size sent by each MTC device may be small, ranging from a few to several hundreds of bytes [2], [3]. One short data packet of MTC devices is made up of information data and metadata, where the latter may be from media-accesscontrol layer and higher layers. Compared to the information data size, the size of metadata, containing various information such as logical addresses, packet initiation and termination, synchronization and security, may not be negligible for MTC devices [4].

MTC devices usually have to meet stringent power consumption requirements. Their battery lifetime is expected to be 10 years or more, especially for MTC devices deployed in extreme conditions or unreachable places [5], [6]. Limited by the cost, chip space and battery capacity of massive MTC devices, the design of energy-efficient MTC networks is an urgent research problem to be addressed. The focus of this paper is power-domain non-orthogonal multiple access (NOMA) [7], in which multiple MTC devices with different power allocations conduct the transmission over the same time-slot and the same frequency channel.

\section{A. State of the Art}

Short packets are the most common data traffic generated by MTC devices. Shannon capacity assumes infinite coding blocklength and sufficiently small decoding error probability, which may not be a suitable tool for analysis the MTC networks [8]. The maximal coding rate of finite blocklength was obtained in [9], which takes the communication channel as a bit pipe of randomly varying size. Based on the rate of finite blocklength, the blocklength-limited performances of different Automatic Repeat-reQuest (ARQ) protocols [10], [11], relay systems [12], [13] and delay sensitive networks [14], [15] have been investigated.

On the one hand, small cell network is a promising technology for $5 \mathrm{G}$ mobile communication systems for a substantial increase of the network capacity and density [16]. However, 
more than $50 \%$ of the energy is consumed by various computation tasks at small cell BSs [17]. Therefore, improving the energy efficiency (EE) of massive MTC networks is becoming an ever-increasingly urgent task to tackle. Joint optimization of computation and communication radio have been investigated to reduce the computation energy consumption in both uplink and downlink systems [18]-[20]. Exploiting the energy harvesting technology or wireless energy and information transmission technology, the MTC devices obtaining energy from other devices to make itself EE transmission in short packet communication was first analyzed in [21], followed by the studies of resource allocation for wireless powered Internet of Things (IoT) with finite blocklength consideration [22], [23]. However, due to the limitation of cost and chip space, many MTC devices may not be able to support wireless powered communication or energy harvesting at this stage. From the perspective of power control, [24]-[26] optimized the transmission power of MTC devices to minimize the energy consumption.

On the other hand, the dilemma between the limited radio resources and the demand for massive connectivity has driven the research of NOMA for massive MTC networks. Compared with orthogonal multiple access (OMA), NOMA and cooperative NOMA enjoy improved network capacity [27], [28]. A new multiple-input multiple-output non-orthogonal multiple access (MIMO-NOMA) scheme has been designed for IoT with small packet transmission in [29], where one user is served with its Quality of service (QoS) requirement strictly being met, and the other user is served opportunistically. Moreover, the trade-off among the transmission rate, energy consumption, decoding error probability and the transmission latency have been investigated for downlink NOMA systems in [30], [31]. To efficiently support massive MTC devices, a multi-layer grant-free NOMA scheme for short packet transmission was proposed in [32].

\section{B. Motivation and Contributions}

Massive MTC networks need to meet diverse QoS requirements in terms of massive connectivity, reliability, EE, latency, throughput, short packets and uplink dominated transmissions. The works of $[10]-[15][22]-[26]$ are mainly focused on OMA, which may not support massive MTC devices in $5 \mathrm{G}$ and beyond for lower spectrum efficiency. Although the authors of [29]-[31] investigated the NOMA performances in short packet communication, they are inapplicable to massive MTC devices in uplink transmissions. The system reliability subject to power and decoding complexity constraints has been studied in [32], however, the resource allocation in uplink NOMAbased short packet communications remains an open issue. To the best of our knowledge, the energy efficient massive MTC networks communicating using short packets is not well investigated.

Against this background, this paper aims to maximize the achievable effective EE in short packet communication for all MTC devices subject to minimum data rate requirements, by taking into account of transmission scheduling technique in uplink NOMA and power control policy. The main contributions of this paper are summarized as follows:
TABLE I

Notations USED In This Paper

\begin{tabular}{ll}
\hline Notions & Meanings \\
\hline $\mathcal{M}, \mathcal{N}$ & Set of MTC devices and subchannels \\
$M, N$ & Number of MTC devices and subchannels \\
$L_{m}$ & Short packet blocklength \\
$N_{n}$ & Maximum number of MTC devices in subchannel $n$ \\
$M_{n}$ & Number of MTC devices in subchannel $n$ \\
$h_{m}, n$ & Channel gain of $m$ th MTC device in $n$th subchannel \\
$p_{m}, n$ & Transmission power of $m$ th MTC device in $n$th subchannel \\
$\varepsilon_{m}, n$ & Effective decoding error probability \\
$P_{m}$ max & Maximum transmission power of each MTC device \\
$x_{m, n}$ & Subchannel allocation index \\
$\mathcal{U}_{n}$ & Set of MTC devices sharing the same subchannel \\
$M_{n}$ & Number of MTC devices sharing the same subcahnnel \\
$\mathbf{p}^{*}, \mathbf{x}^{*}$ & Optimal power/subchannel allocation matrix \\
$\eta$ & Energy efficiency \\
$\omega, \varphi, \epsilon$ & Learning rate, discount factor and exploration probability \\
$\lambda, \mu$ & Lagrange multipliers \\
$\rho, \tau$ & SINR and power amplifier \\
$\theta_{i}, i=1,2,3$ & Convergence error in Algorithm 1 \\
$Q^{-1}(\cdot)$ & Inverse of Gaussian Q-function \\
MTC & Machine-type Communications \\
HTC & Human-type Communication \\
OMA & Orthogonal Multiple Access \\
NOMA & Non-Orthogonal Multiple Access \\
EE & Energy Efficiency \\
BPCU & Bit Per Channel Use \\
QoS & Quality of Service \\
IoT & Internet of Things \\
\hline & \\
\end{tabular}

1) Focusing on uplink NOMA-based massive MTC networks, we define the achievable effective EE for all the MTC devices by considering short packet communication characteristics and the decoding error probability at the receiver. Constrained by minimum data rate requirements of MTC devices, maximum number of MTC devices in each subchannel and the maximum transmission power of MTC devices, we maximize the achievable effective EE via subchannel allocation and power allocation two decoupled subproblems.

2) We model the formulated subchannel allocation problem as a multi-agent Markov decision process. The central controller is employed to manage the subchannel allocations, and each selfish and rational MTC device acts as a learning agent to submit its requests to the center controller. We then propose an efficient Q-learning based subchannel allocation for maximizing the achievable effective EE.

3) Given the subchannel allocation, we transform the fractional programming problem on the achievable effective EE into a series of subproblems based on the Dinckel Algorithm [33]. By exploiting the first order Taylor approximation, we approximate the achievable effective rate of MTC devices in uplink NOMA-based short packet communications and obtain its lower bound. Moreover, we obtain the optimal transmission power of each MTC device with the Lagrangian-dual algorithm.

4) Compared with existing schemes, the results indicate that the proposed algorithm can significantly improve the achievable effective EE of MTC devices in short packet communications while guaranteeing their QoS requirements. 
The rest of this paper is organized as follows. In Section II, we introduce the short packet communication in uplink NOMA-based massive MTC networks. Section III formulates the optimization problem of achievable effective EE. Section IV presents the reinforcement learning based subchannel allocation and solution of power allocation scheme is given Section V. Simulation results and conclusions are presented in Section VI and VII, respectively. Notations used in this paper are listed in Table I.

\section{SySTEM MODEL}

\section{A. Uplink NOMA-based Massive MTC Networks}

We consider an uplink NOMA-based massive MTC networks scenario as shown in Fig. 11(a), where $\mathcal{M}=$ $\{1,2, \ldots, M\}$ MTC devices in the active mode transmit data to BS. The available bandwidth $W$ is divided into $N$ orthogonal subchannels. The set of subchannel is denoted by $\mathcal{N}=\{1,2, \ldots, N\}$, and they are employed to serve the active MTC devices. Furthermore, we assume that each MTC device is allowed to use at most one subchannel. The set of MTC devices sharing the $n$th subchannel is denoted as $\mathcal{U}_{n}$ and the number of MTC devices is $M_{n}=\left|\mathcal{U}_{n}\right|$, $M=M_{1}+M_{2}+\ldots+M_{N}$. If $M_{n}>1$, the $n$th subchannel is a NOMA link, where $M_{n}$ MTC devices simultaneously transmit data to BS over this link, and the receiver needs to employ multi-user decoding technology to separate the overlap signals. Otherwise, it belongs to an OMA link and the assigned MTC device will use the subchannel without multiuser interference. The scheme that different number of MTC devices accommodated on different subchannels is a special case of hybrid NOMA [7]. To balance the performance and complexity in multiple subchannel NOMA, we assume that the maximum number of MTC devices sharing the $n$th subchannel is limited to $N_{n}$.

Each MTC device transmits with a short packet of $L_{m}$ payload bits including $l_{m}$ bits information data and $l_{0}$ bits additional metadata (such as preamble and header), the structure of which is shown in Fig. 1 (b). The block fading channel model is adopted, in which the fading gain keeps constant in each time slot. The channel gain from the $m$ th MTC device to the BS in the $n$th subchannel is $h_{m, n}$, which is characterized by large scale pathloss of distance and small scale Rayleigh fading. Furthermore, we assume that MTC devices can obtain their own channel gains with feedback. The received signals at the $\mathrm{BS}$ on the $n$th subchannel can be written as

$$
y_{n}=\sum_{m=1}^{M_{n}} \sqrt{p_{m, n}} h_{m, n} s_{m, n}+N_{o},
$$

where $s_{m, n}$ denotes the transmitted signal of the $m$ th MTC device over the $n$th subchannel, and its power denoted as $\mathbb{E}\left(\left|s_{m, n}\right|^{2}\right)=1 . p_{m, n}$ is the transmission power of the $m$ th MTC device over the $n$th subchannel, which is constrained by $p_{m, n} \leq P_{\max } . N_{o}$ represents the noise power per sub-channel.

In the uplink, all the received signals at BS are desired signals, which can be decoded by successive interference cancellation (SIC). However, because of the limited detection ability and the impact of modulation and decoding schemes, the fact that decoding error probability cannot be neglected in
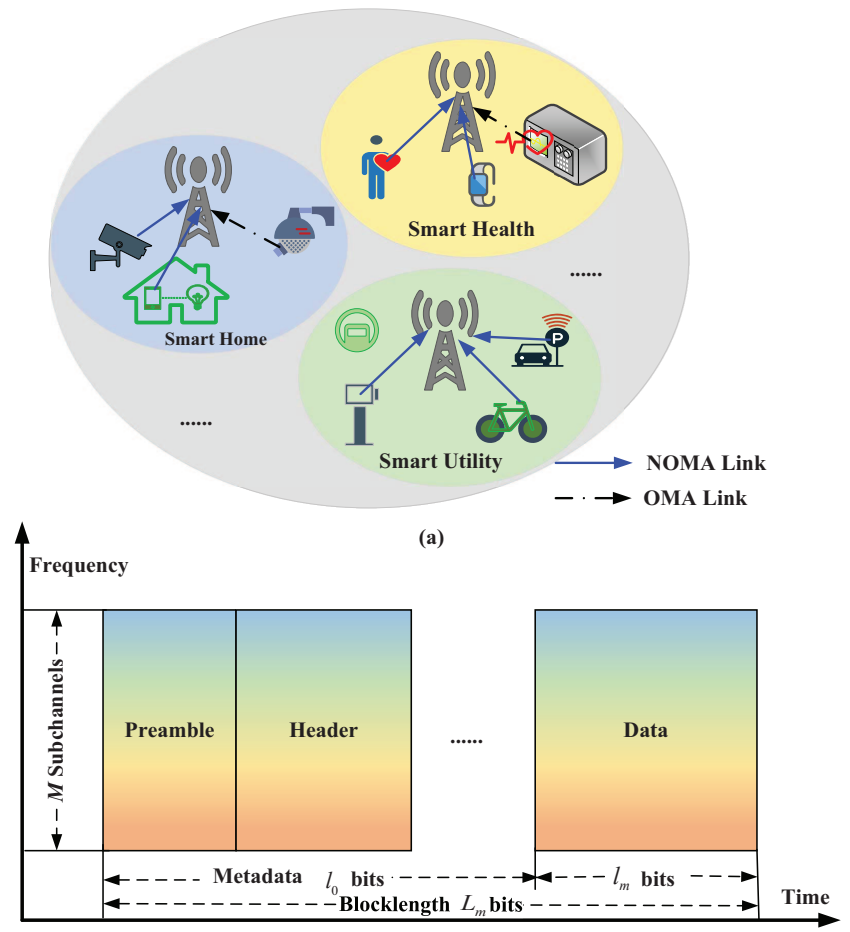

(b)

Fig. 1. (a) Uplink NOMA-based massive MTC Networks System Model; (b) Short Packet Blocklength Structure

short packet communications. The perfect SIC assumption can not be valid as that in Shannon Capacity, due to its assumption of infinite blocklength and free of decoding error. If two or more MTC devices select the same subchannel, the SIC decoding error in the first user will surely cause an error in the second. Therefore, taking both decoding error probability and the SIC decoding error probability in previous step into consideration of $\varepsilon_{m, n}$, the effective decoding error probability $\varepsilon_{m, n}$ can be expressed as

$$
\varepsilon_{m, n}=\prod_{i=1}^{m-1}\left(1-\varepsilon_{i, n}\right) \bar{\varepsilon}_{m, n}+\left[1-\prod_{i=1}^{m-1}\left(1-\varepsilon_{i, n}\right)\right] \tilde{\varepsilon}_{m, n},
$$

which means that the decoding error probability of the $m$ th user in the $n$th subchannel is $\bar{\varepsilon}_{m, n}$ if the former $(m-1)$ stronger MTC devices all decoding successful, otherwise the decoding error probability is $\tilde{\varepsilon}_{m, n}$.

\section{B. Achievable Effective Rate with Short Packet Communica- tion}

Different from downlink NOMA that the decoding order is always fixed, the decoding order is arbitrary in BS because the received signals are all desired. Many factors may influence the decoding order in the BS. For example, the network, channel gain, available resource conditions and QoS constraints [34]-[36]. However, the decoding order in short packet communications is still an open issue and little works have been paid on attention to it. In this work, we assume that $\left|h_{1, n}\right|^{2} \geq \cdots \geq\left|h_{m, n}\right|^{2} \cdots \geq\left|h_{M_{n}, n}\right|^{2}$, and the MTC device with higher channel gains will be decoded earlier at BS, although they may suffer from interference of other 
undecoded MTC devices [7], [20], [37]. Moreover, since the linear function can effectively model the relationship between the residual interference and the power of the received signal [38], [39], we model the residual interference from the first decoded $m-1$ MTC devices in the $n$th subchannel as follows

$$
\begin{aligned}
I_{m, n}^{S I C} & =\sum_{j=1}^{m-1}\left|h_{j, n}\right|^{2} p_{j, n}\left|x_{j, n}-\tilde{x}_{j, n}\right|^{2} \\
& =\sum_{j=1}^{m-1} \varrho_{j, n} p_{j, n}\left|h_{j, n}\right|^{2},
\end{aligned}
$$

where $x_{j, n}-\tilde{x}_{j, n}$ means the difference between the actual signal $x_{j, n}$ and the estimated signal $\tilde{x}_{j, n}$, and $\varrho_{j, n}=$ $\mathbb{E}\left[\left|x_{j, n}-\tilde{x}_{j, n}\right|^{2}\right]$. Specifically, $\varrho_{j, n}=0$ means perfect SIC, $0<\varrho_{j, n}<1$ denotes imperfect SIC and $\varrho_{j, n}=1$ indicates no SIC.

Then, the channel gain-to-interference-plus-noise ratio (CINR) for the $m$ th user in the $n$th subchannel can be expressed as

$$
\gamma_{m, n}=\frac{\left|h_{m, n}\right|^{2}}{I_{m, n}^{S I C}+\sum_{i=m+1}^{M_{n}} p_{i, n}\left|h_{i, n}\right|^{2}+\sigma^{2}},
$$

where $\sum_{i=m+1}^{M_{n}} p_{i, n}\left|h_{i, n}\right|^{2}$ is the interference of the $m$ th MTC device suffering from the $n$th subchannel.

According to [9], the achievable channel coding rate of short packet communications depends on the channel condition, finite blocklength and decoding error probability. Therefore, given the finite blocklength $L_{m}$ and effective decoding error probability $\varepsilon_{m, n}$ of the $m$ th MTC device in the $n$th channel, the achievable instantaneous rate in bits per channel use (BPCU) can be approximated as

$$
r_{m, n}=C_{m, n}-\sqrt{\frac{V_{m, n}}{L_{m}}} \frac{Q^{-1}\left(\varepsilon_{m, n}\right)}{\ln 2},
$$

where

$$
\begin{aligned}
& C_{m, n}=\log _{2}\left(1+p_{m, n} \gamma_{m, n}\right), \\
& V_{m, n}=1-\frac{1}{\left(1+p_{m, n} \gamma_{m, n}\right)^{2}} .
\end{aligned}
$$

$V_{m, n}$ is the channel dispersion measuring the stochastic variability of the channel relative to a deterministic channel with the same capacity [9]. $Q^{-1}($.$) is the inverse of Gaussian$ Q-function $Q(x)=\int_{x}^{\infty} \frac{1}{\sqrt{2 \pi}} e^{-\frac{t^{2}}{2}} d t$. For simplicity, we let $\kappa_{m, n}=\frac{Q^{-1}\left(\varepsilon_{m, n}\right)}{\ln 2 \sqrt{L_{m}}}$. The expression in 5a means that compared with Shannon Capacity, achieving the targeted decoding error probability $\varepsilon_{m, n}$ incurs a penalty term on the achievable rate for MTC devices in the short packet communications.

Considering the trade-off between error probability and transmission rate, we adopt the effective rate [30] as the metric to for performance evaluation of massive MTC networks. The effective rate measured in bits per $L_{m}$ channel use is given by

$$
R_{m, n}=\left\{\begin{array}{cc}
0, & \varepsilon_{m, n}, \\
L_{m} r_{m, n}, & 1-\varepsilon_{m, n} .
\end{array}\right.
$$

where the effective rate $R_{m, n}$ in bits per $L_{m}$ channel use is zero when the information fails to be decoded, or the effective rate $R_{m, n}$ in bits per $L_{m}$ channel use equals to $L_{m} r_{m, n}$.
Taking the expectation of the effective rate $R_{m, n}$ divided by $L_{m}$ with respect to the decoding error probability, the achievable effective rate in BPCU can be expressed as

$$
\bar{R}_{m, n}=\mathbb{E}_{\varepsilon_{m, n}}\left[\frac{R_{m, n}}{L_{m}}\right]=\left(1-\varepsilon_{m, n}\right) r_{m, n} .
$$

\section{Maximization of Achievable EfFective Energy EFFICIENCY}

Due to the limited power and the requirement of long battery lifetime, MTC devices are required to transmit information data in an energy efficient manner. In contrast to existing studies, we aim to maximize the achievable effective EE, which is defined as the total achievable effective rate with unit power consumption per channel use. Thus, we formulate the system achievable effective EE as a ratio of the total achievable effective rate per channel use to the total power consumption (BPCU/Joule), which is expressed as

$$
\eta_{E E}=\frac{\sum_{n=1}^{N} \sum_{m=1}^{M_{n}} \bar{R}_{m, n}}{P_{\text {total }}},
$$

where $P_{\text {total }}$ is the total power consumption of all MTC devices and expressed as $\sum_{n=1}^{N}\left(\sum_{m=1}^{M_{n}}\left(\frac{1}{\tau} p_{m, n}+P_{m, C}\right)\right)$. $P_{m, C}$ is the static circut power consumption, which also plays a significant role in EE [20]. $\tau \in[0,1]$ is a constant value which accounts for the power amplifier efficiency.

The achievable effective EE maximization problem is formulated as follows

$$
\begin{aligned}
\max _{\mathbf{p}, \mathbf{x}} & \eta_{E E} \\
\text { s.t. } \quad & r_{m, n} \geq R_{m, \min }, \forall m, n, \\
& \sum_{n=1}^{N} x_{m, n} p_{m, n} \leq P_{\max }, \forall m, \\
& \sum_{m=1}^{M} x_{m, n} \leq N_{n}, \forall m, \\
& x_{m, n}\left(x_{m, n}-1\right)=0, \forall m, n .
\end{aligned}
$$

where $9 \mathrm{~b}$ represents the minimal transmission rate requirement of each MTC device, $x_{m, n}$ denotes whether the $n$th subchannel is assigned to the $m$ th MTC device, i.e., $x_{m, n}=1$ if the $m$ th MTC device selects the $n$th subchannel to transmit, or $x_{m, n}=0$. (9d) means that the number of MTC devices sharing the same subchannel is limited by an upper bound, i.e. $M_{n} \leq N_{n}$, 9e guarantees the $x_{m, n}$ is a binary number,

However, the introduced multiuser interference couples all the MTC devices sharing same subchannels, which makes the objective function in $\mathbf{P 1}$ a ratio between a nonconvex function and an affine function. In addition, the subchannel indicator is binary variable. P1 is classified into a nonconvex mixed integer nonlinear problem (MINLP) with fractional programming, which is a nonpolynomial problem and difficult to solve. Hence, with the given subchannel and power allocation, we reformulate the short packet communication rate in $(5)$ as

$$
f(\rho)=\log _{2}(1+\rho)-\kappa \frac{\sqrt{\rho(\rho+2)}}{1+\rho},
$$

where $\rho=p \gamma$ is the SINR. Due to the convexity of the second term in 10 is not known, the convexity of $f(\rho)$ cannot 
directly obtain. In the following, we first give the convexity analysis of $f(\rho)$, then exploit it to design subchannel and power allocation algorithms.

Lemma 1. The convexity of the short packet communication rate $f(\rho)$ is analyzed as follows:

- $f(\rho)$ is a concave function under 1$) \rho \geq g^{-1}(\kappa)$ if $\kappa \geq \vartheta$ and 2) $\rho \geq g_{2}^{-1}(\kappa)$ if $\kappa \leq \vartheta$;

- $f(\rho)$ is a convex function under $g^{-1}(\kappa) \leq \rho \leq g_{2}^{-1}(\kappa)$ if $\kappa \leq \vartheta$.

where $g(\rho)=\frac{\log _{2}(1+\rho)(1+\rho)}{\sqrt{\rho(\rho+2)}}, g_{2}(\rho)=\frac{(1+\rho)[\rho(\rho+2)]^{\frac{3}{2}}}{\ln 2\left(3 \rho^{2}+6 \rho+1\right)}, \vartheta=$ $g_{2}\left(\rho_{0}\right)=g\left(\rho_{0}\right)$ and $\rho_{0}=0.6904$.

Proof: Refer to Appendix A.

Based on Lemma 1, the short packet communication rate is a concave function if SINR is sufficiently large, i.e. $\rho>$ $g_{2}^{-1}(\kappa)$ and $\rho>g^{-1}(\kappa)$, and the achievable rate increases with the SINR. To maximize the achievable rate under QoS requirements, we obtain Proposition 1.

Proposition 1. In a massive MTC system with target decoding error probability $\varepsilon \geq 10^{-5}$ and blocklength $L_{m} \geq 392$, the rate $f(\rho)$ is concave and increasing with $\rho$ if $\rho>-5 d B$.

Proof: Based on Lemma 1, we have $f^{\prime \prime}(\rho)<0$ if $\phi(\rho)<$ 0 , where $\phi(\rho)=-(1+\rho)+\frac{\kappa}{[\rho(\rho+2)]^{\frac{3}{2}}}+\frac{3 \kappa}{\sqrt{\rho(\rho+2)}}$. We can obtain $\phi(\rho)$ is descreasing in $\rho, L$ and $\varepsilon$. When $\rho=-5 d B$, $L=392$, and $\varepsilon=10^{-5}, \phi(\rho)=-1.8168 \times 10^{-4}$ and $f(\rho)>$ $0, f^{\prime}(\rho)>0$. Hence, the rate $f(\rho)$ is concave and increasing with the $\rho$ if $\rho>-5 d B$.

\section{REINFORCEMENT LEARNING BASED SUbCHANNEL ALLOCATION}

In the considered massive MTC network scenario, we model the process of subchannel allocation as a multi-agent finite Markov decision process. A central controller is employed to manage the subchannel allocation and each MTC device, with selfish and rational, acts as a learning agent to submit their requests to the central controller. Furthermore, the multiagent Q-learning scheme for NOMA based massive MTC networks is proposed to solve the subchannel allocation. The key components of multi-agent Q-learning framework for NOMA based massive MTC networks are given in Fig. 2. Each subchannel allocation solution corresponds to an action taken by the central controller. In the process, the rewards of MTC devices depend on the states and actions of both themselves and central controller. The state, action, transition probability and reward are defined as following:

State: An MTC device is in state $s_{n}$ if it occupies the $n$th sub-channel.

Actions: The action $a\left(s, s^{\prime}\right)$ is defined as a transition from a certain state $s$ to a target stat $s^{\prime}$, which is a request of MTC device to change its preference from one subchannel to another subchannel. When the $m$ th MTC device needs to be served, it will send an action request to the central controller according to the current state $s$ and $\{\mathcal{U}\}$. If the number of MTC device sharing this subchannel is equal to the maximum number $N_{n}$, the action is unavailable and set as $0\left(x_{m, n}=0\right)$; otherwise

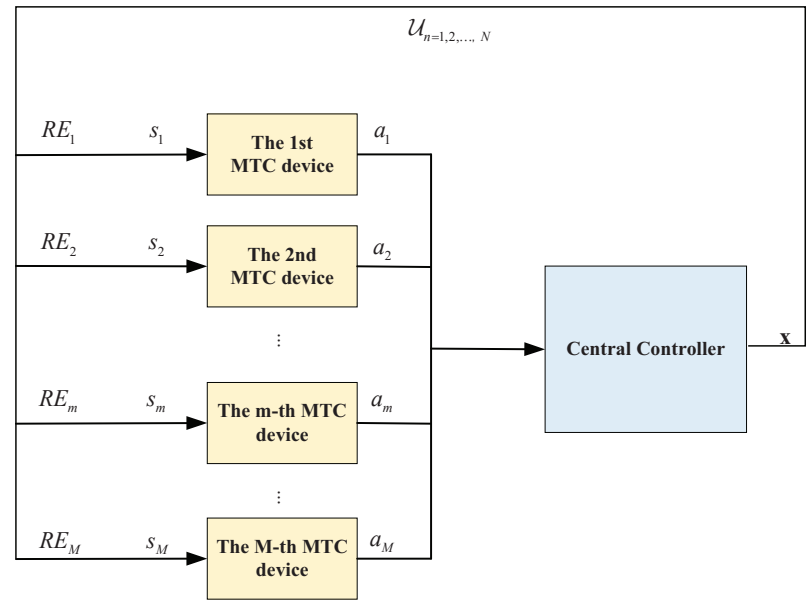

Fig. 2. Multi-agent Q-learning Frame for NOMA based mMTC Networks

it will be 1 (the subchannel allocation temporarily is set as $\left.x_{m, n}=1\right)$. In summary, we have

$$
a\left(s, s^{\prime}\right)= \begin{cases}1 & \left|\mathcal{U}_{n}\right|<N_{n}, \\ 0 & \left|\mathcal{U}_{n}\right|=N_{n} .\end{cases}
$$

Transition Probability: The transition probability $\pi_{a}$ denotes the probability of an MTC device choosing a certain action $a$, and resulting in a transition from state $s$ to $s^{\prime}$. However, they do not know other MTC devices' preferences. With reinforcement learning, the MTC devices learn what action to take in the next step according to the action selection policy.

Reward: The reward $R E(s, a)$ is obtained by the $m$ th MTC device after its request is accepted by the central controller. No reward is given if the request is blocked, which means that the $m$ th MTC device cannot use its requested subchannel. As defined in (8), the achievable effective EE is related to the achievable effective rate in the numerator and the total transmission power in the denominator. Fixing the power allocation and other MTC devices' $\{\mathcal{M}-m\}$ subchannel allocation, the achievable effective EE of the $m$ th MTC device is concave and increasing with CINR if $\rho>-5 \mathrm{~dB}$. To reduce the computation complexity of central controller, the reward $R E(s, a)$ is defined as the CINR of the requested subchannel.

At each selection time, the MTC device receives a reward and passes into the successor state $s^{\prime}$. The selection of actions depends on a policy. The goal of MTC device is to find an optimal policy to decide which subchannel is its optimal choice. The optimal policy $\pi_{\text {opt }}(s)=\max _{a \in \mathcal{A}} Q(a, s)$, where $Q(a, s)$ is the action-utility function and represents the expected discounted reward when starting in state $s$ and selecting action $a$.

Since the Q-values provide insights on the future quality of the actions in the successor state, the update rule of Q-value function for state-action pair [40] is

$$
\begin{aligned}
Q(a, s) & =Q(a, s) \\
& +\omega\left(R E(a, s)+\max _{a \in \mathcal{A}} Q\left(a^{\prime}, s^{\prime}\right)-Q(a, s)\right),
\end{aligned}
$$


where $\omega$ represents the learning rate and $\varphi$ is the discount factor to balance the immediate and future reward.

Furthermore, MTC devices make the decision whether exploration or exploitation to maximize the rewards at each decision epoch. We consider the $\epsilon$-greedy exploration. The MTC device selects exploration with probability $\epsilon$ to discover new state and submits a new subchannel request to central controller. Moreover, it will select exploitation with $1-\epsilon$ to select the same action without submiting any suchannel requests and use previous subchannel allocation policy. When the learning time increases, MTC devices will choose smaller exploration probability to maximize its reward. Thus, the exploration probability $\epsilon(s)$ can be written as

$$
\epsilon_{t_{q}+1}(s)=\delta \epsilon_{t_{q}}(s)
$$

where $\delta$ is the ratio of the number of current state to the maximum learning times.

\section{Power Allocation for Maximizing Achievable EFFECTIVE EE}

Since the problem in (9) belongs to a fractional problem, its optimal solution is non-trival. Without loss of generality, we define the maximum achievable effective EE of P1 as $\eta^{*}$, and

$$
\begin{aligned}
\eta^{*} & =\frac{\sum_{n=1}^{N} \sum_{m=1}^{M_{n}} \bar{R}_{m, n}\left(\mathbf{p}^{*}\right)}{P_{\text {total }}\left(\mathbf{p}^{*}\right)}, \\
& =\max _{\left\{p_{m, n}\right\}} \frac{\sum_{n=1}^{N} \sum_{m=1}^{M_{n}} \bar{R}_{m, n}}{P_{\text {total }}} .
\end{aligned}
$$

However, due to the channel dispersion in short packet communications, $r_{m, n}$ is not always concave/convex, meaning that the optimization problem in 9 is a non-convex optimization problem due to the non-concavity involed in the objective function. According to the analysis in Appendix A, $\log _{2}(1+\rho)$ and $\kappa \frac{\sqrt{\rho(\rho+2)}}{1+\rho}$ are continuous concave functions. Hence, we exploit the first-order Taylor approximation to approximate $r_{m, n}$, which is expressed as

$$
\begin{aligned}
r_{m, n} & =C_{m, n}-\kappa_{m, n} f_{1}\left(p_{m, n}^{(k)}, \gamma_{m, n}\right) \\
& -\kappa_{m, n} f_{2}\left(p_{m, n}^{(l)}, \gamma_{m, n}\right)\left(p_{m, n}-p_{m, n}^{(l)}\right),
\end{aligned}
$$

where $f_{1}(p, \gamma)=\frac{\sqrt{p \gamma(p \gamma+2)}}{1+p \gamma}, f_{2}(p, \gamma)=\frac{\gamma}{(1+p \gamma)^{2} \sqrt{p \gamma(p \gamma+2)}}$ is the first order partial derivative of $f_{1}(p, \gamma)$ in terms of $p$. Both $f_{1}\left(p_{m, n}^{(l)}, \gamma_{m, n}\right)$ and $f_{2}\left(p_{m, n}^{(l)}, \gamma_{m, n}\right)$ are constant value in each iteration $l$.

Furthermore, MTC devices sharing the same subchannel are coupled through intra-interference, rendering $r_{m, n}$ still being a quasi-concave function with respect to $p_{m, n}$. The numerator of objective function in (14) is a summation of quasi-concave functions, which invalidates the theory of nonnegative summation of concave functions preserving concavity for quasi-concave functions [41]. By exploiting the successive convex approximation [42], we first establish a concave lower bound of $C_{m, n}$, which is parameterized by a given power allocation $\mathbf{p}$,

$$
\begin{aligned}
C_{m, n} & \geq \alpha_{m, n} \log _{2}\left(p_{m, n} \gamma_{m, n}\right)+\beta_{m, n} \\
& =\alpha_{m, n}\left[\log _{2}\left(p_{m, n}\right)+\log _{2}\left(\gamma_{m, n}\right)\right]+\beta_{m, n},
\end{aligned}
$$

where

$$
\begin{aligned}
\alpha_{m, n} & =\frac{\tilde{p}_{m, n} \tilde{\gamma}_{m, n}}{1+\tilde{p}_{m, n} \tilde{\gamma}_{m, n}}, \\
\beta_{m, n} & =\log _{2}\left(1+\tilde{p}_{m, n} \tilde{\gamma}_{m, n}\right)-\alpha_{m, n} \log _{2}\left(\tilde{p}_{m, n} \tilde{\gamma}_{m, n}\right) .
\end{aligned}
$$

the equality in 16 holds when $p_{m, n} \gamma_{m, n}=\tilde{p}_{m, n} \tilde{\gamma}_{m, n}$.

Furthermore, we let $q_{m, n}=\log _{2}\left(p_{m, n}\right)$, the lower bound of $C_{m, n}$ becomes

$$
\tilde{C}_{m, n}=\alpha_{m, n}\left[q_{m, n}+\log _{2}\left(\tilde{\gamma}_{m, n}\right)\right]+\beta_{m, n},
$$

where $\tilde{C}_{m, n}$ is a concave function [42], and

$\tilde{\gamma}_{m, n}=\frac{\left|h_{m, n}\right|^{2}}{\sum_{j=1}^{m-1} \varepsilon_{j, n} 2^{q_{j, n}}\left|h_{j, n}\right|^{2}+\sum_{i=m+1}^{M_{n}} 2^{q_{i, n}}\left|h_{i, n}\right|^{2}+\sigma^{2}}$.

Applying the equation of both (15) and (17) into (7), the $\bar{R}_{m, n}$ can be rewritten as

$$
\begin{aligned}
\bar{R}_{m, n} & =\left(1-\varepsilon_{m, n}\right)\left[\alpha_{m, n}\left(q_{m, n}+\log _{2} \tilde{\gamma}_{m, n}\right)+\beta_{m, n}\right] \\
& -\left(1-\varepsilon_{m, n}\right)\left[\kappa_{m, n} f_{1}\left(p_{m, n}^{(l)}, \gamma_{m, n}\right)\right] \\
& -\left(1-\varepsilon_{m, n}\right) \kappa_{m, n} f_{2}\left(p_{m, n}^{(l)}, \gamma_{m, n}\right)\left(p_{m, n}-p_{m, n}^{(l)}\right) .
\end{aligned}
$$

Theorem 1. The maximum achievable effective EE can be achieved only when $\eta^{*}$ and the optimal resource allocation policies $\mathbf{p}^{*}$ satisfy,

$$
\begin{aligned}
\underset{\left\{p_{m, n}\right\}}{\max } & \sum_{n=1}^{N} \sum_{m=1}^{M_{n}} \bar{R}_{m, n}-\eta^{*} P_{\text {total }} \\
& =\sum_{n=1}^{N} \sum_{m=1}^{M_{n}} \bar{R}_{m, n}\left(\mathbf{p}^{*}\right)-\eta^{*} P_{\text {total }}\left(\mathbf{p}^{*}\right) \\
& =0 .
\end{aligned}
$$

Proof: Refer to Appendix B.

Based on the Dinckel Algorithm [33], we propose an iterative power allocation algorithm to maximize the achievable effective EE in (19), which is shown in Algorithm 1. The proposed power allocation algorithm converges to its optimum when a fixed convergence error $\theta_{3}$ or the maximum of iterations is achieved. Furthermore, we convert it to a series of subproblems as follows

$$
\begin{aligned}
\text { (P2) } \max _{\mathbf{p}} & \sum_{n=1}^{N} \sum_{m=1}^{M_{n}} \bar{R}_{m, n}-\eta^{(t-1)} P_{\text {total }} \\
\text { s.t. } & 9 b-9 c||_{p_{m, n}=2^{q m, n}, \forall m, n .}
\end{aligned}
$$

where $\eta^{(t-1)}$ is a non-negative parameter.

Lemma 2. Problem $\boldsymbol{P 2}$ is a concave function problem, any of its local maximum is a global maximum and Karush-KuhnTucker (KKT) conditions are sufficient to maximum.

Proof: According to [42] and the characteristic of the first-order Taylor approximation, the $\bar{R}_{m, n}$ in 18 can be proved to be concave in $\mathbf{q}$ due to the fact that the log-sumexp function convex. It means that the first part of objective 


$$
p_{m, n}=\frac{\left(1-\varepsilon_{m, n}+\mu_{m, n}\right) \alpha_{m, n}}{\ln 2\left[\kappa_{m, n}\left(1-\varepsilon_{m, n}+\mu_{m, n}\right) f_{2}\left(p_{m, n}^{k}, \gamma_{m, n}^{k}\right)+\mathrm{I}_{m, n}^{\text {inter }}+\mathrm{I}_{m, n}^{r e s}+\eta / \tau\right]},
$$

where

$$
\begin{aligned}
& \mathrm{I}_{m, n}^{\text {inter }}=\sum_{j=1}^{m-1}\left(1-\varepsilon_{j, n}+\mu_{j, n}\right) \alpha_{j, n} \frac{\gamma_{j, n}\left|h_{m, n}\right|^{2}}{\left|h_{j, n}\right|^{2}}, \\
& \mathrm{I}_{m, n}^{r e s}=\sum_{i=m+1}^{M_{n}}\left(1-\varepsilon_{i, n}+\mu_{i, n}\right) \alpha_{i, n} \varrho_{m, n} \frac{\gamma_{i, n}\left|h_{m, n}\right|^{2}}{\left|h_{i, n}\right|^{2}} .
\end{aligned}
$$

function in (18) is concave in $\mathbf{q}$ and the feasible set in (9b). Furthermore, it can be easily proven that the power consumption of the first part in objective function (18) and the transmission power set in $9 \mathrm{c}$ are also convex in $\mathbf{q}$. Therefore, we conclude that the problem $\mathbf{P 2}$ is a concave function. This implies that any local maximum is a global maximum and KKT conditions are sufficient to the maximum solution.

\section{A. Proposed Solution}

Since $\mathbf{P 2}$ is a standard concave maximization problem, we derive an algorithm to solve this concave problem in this section using gradient methods, which are computationally efficient and without the need for a brute-force or heuristic search of any kind. In uplink NOMA, we can first obtain the solution of the user with the lowest channel gain, which is free of interferences. Then, we can obtain the solution of the user with the second lowest channel gain. At last, we can obtain the solution of the user with the highest channel gain. Once the $\mathbf{q}$ of all MTC devices are obtained, we can transform back to the transmission power $P$-space with $p_{m, n}=2^{q_{m, n}}$.

We derive an algorithmic solution to $\mathbf{P 2}$ via the dual problem $\min _{\mu, \lambda \geq 0} Q(\mu, \lambda)$, where $\mu$ and $\lambda$ are the Lagrange multipliers of $9 \mathrm{~b}$ ) and $(9 \mathrm{c}$. The dual function is given by

$$
Q(\mu, \lambda)=\max _{\mathbf{q}} L(\mathbf{q}, \mu, \lambda),
$$

and the corresponding Lagrangian is

$$
\begin{aligned}
& L(\mathbf{q}, \mu, \lambda)=\sum_{n=1}^{N} \sum_{m=1}^{M_{n}}\left(1-\varepsilon_{m, n}+\mu_{m, n}\right) \tilde{r}_{m, n} \\
& -\eta\left(\sum_{n=1}^{N} \sum_{m}^{M_{n}} \tau 2^{q_{m, n}}+\sum_{n=1}^{N} \sum_{m}^{M_{n}} p_{m, n, C}\right) \\
& -\sum_{n=1}^{N} \sum_{m=1}^{M_{n}} \mu_{m, n} R_{m, \text { min. }} .
\end{aligned}
$$

Since the Lagrangian 22 is strictly concave in $\mathbf{q}$, it has a unique solution. Furthermore, because the dual function $Q(\mu, \lambda)$ is differentiable everywhere and we can employ the gradient-descent to solve the outer minimization

$$
\mu_{m}^{(s+1)}=\left[\mu_{m}^{(s)}+\xi_{\mu}\left(R_{m, \min }-\sum_{n=1}^{N} \bar{R}_{m, n}\right)\right]^{+}
$$

where $\xi_{\mu}$ is sufficiently small step sizes, $[x]^{+}=\max (0, x)$ and $s$ is an iteration number.

The dual function (21) is evaluated by finding the stationary point of the Lagrangian (22) with multipliers fixed. With KKT conditions, we obtain the optimal transmission power in (24).

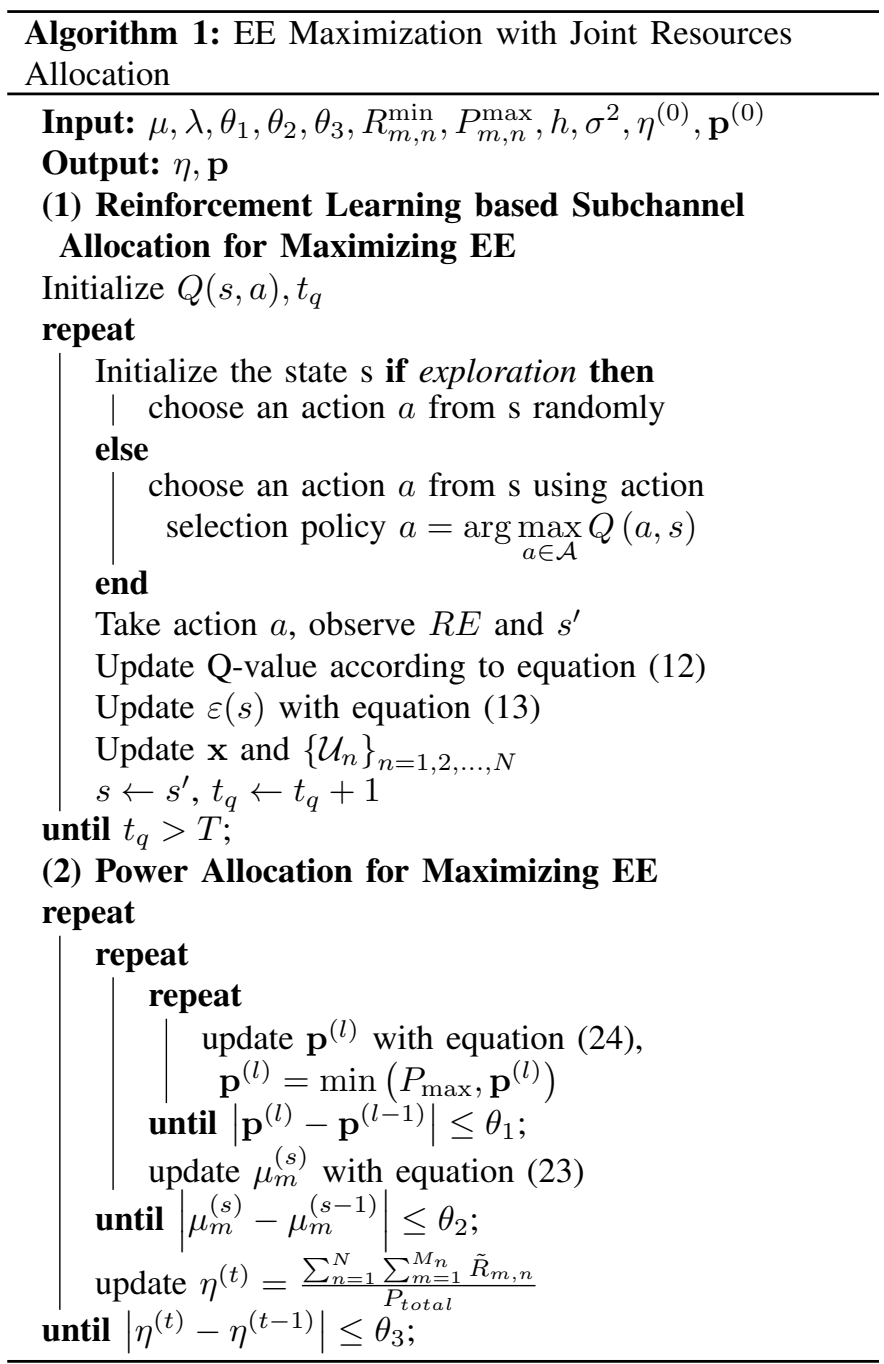

\section{B. Complexity and Convergence}

The computational complexity of Algorithm 1 is the total computational complexity of the proposed scheme, which includes the computational complexity of reinforcement learning based subchannel allocation and that of power allocation for maximizing achievable effective EE. Because there are $M$ MTC devices and $N$ subchannels, the complexity of subchannel allocation is $\mathcal{O}(M N)$ Q-learning iteration. The complexity of sublinear rate, such as $f\left(x_{k}\right)-f^{*} \leq \xi$, is $\mathcal{O}\left(\frac{1}{\xi^{2}}\right)$. Therefore, the complexity of power allocation algorithm is $\mathcal{O}\left(\frac{M N}{\theta_{1}^{2}}\right) \mathcal{O}\left(\frac{M}{\theta_{2}^{2}}\right) \mathcal{O}\left(\frac{1}{\theta_{3}^{2}}\right)$. The computational complexities 
TABLE II

Simulation Parameters [4]

\begin{tabular}{|l|l|}
\hline Parameter & Settings \\
\hline Transmission bandwidth & $10 \mathrm{MHz}$ \\
\hline Number of subchannels & 50 \\
\hline Bandwidth of one subchannel & $180 \mathrm{kHz}$ \\
\hline Distance dependent path loss & $\begin{array}{l}128.1+37.6 \log 10(\mathrm{~d}) \\
(\mathrm{dB}), \mathrm{d}(\mathrm{km})\end{array}$ \\
\hline Shadowing standard deviation & $8 \mathrm{~dB}$ \\
\hline Fading & Rayleigh fading \\
\hline Noise Density & $-174 \mathrm{dBm} / \mathrm{Hz}$ \\
\hline Max MTC devices in same subchannel $N_{n}$ & 2 \\
\hline Max transmission power of MTC $P_{m}, \max$ & $23 \mathrm{dBm}$ \\
\hline Static circuit power $P_{m, \mathrm{C}}$ & $5 \mathrm{dBm}$ \\
\hline Power amplifier efficiency $\tau$ & 0.38 \\
\hline Blocklength $L_{m}$ & 392 \\
\hline Decoding error probability $\varepsilon$ & $10^{-5}$ \\
\hline
\end{tabular}

increase with the number of MTC devices.

\section{Vi. Performance Evaluations}

In this section, we evaluate the proposed achievable effective EE performance in short packet communication for uplink NOMA-based massive MTC networks through Monte Carlo simulations. We assume that there are $N_{n}$ users simultaneously multiplexed on the same subchannel to perform short packet communication. The system parameters used in simulations are given in Table II unless otherwise stated, where the wireless parameters are based on the 3GPP standard in [43]. Considering the characteristics of MTC devices, we set the static circuit power of MTC devices to $5 \mathrm{dBm}$. As defined in [44], the packet size follows 20 200 Pareto distribution in massive MTC devices scenario, and the higher layer protocol overhead is 29 bytes. Therefore, we set the blocklength range is $392 \sim 1832$ symbols. Although the target packet error ratio (PER) of an individual massive MTC transmission is on the order of $10^{-1}$ [45], we consider a lower PER as $10^{-5}$, which can cover most MTC devices, such as mission critical MTC devices and low-cost MTC devices.

We name the proposed maximizing achievable EE algorithm applied to uplink NOMA-based massive MTC networks with short packet communication as maxEE-NOMA-proposed in Fig. 3, Fig. 4 and Fig. 5. For convenience, we give the explanation of the compared schemes and other baseline schemes:

1) To study the performance of short packet communication in uplink NOMA, we name the proposed maximizing achievable EE algorithm applied to a conventional Orthogonal Frequency Division Multiple Access (OFDMA)system as maxEE-OFDMA, where each user can only be assigned to one subchannel.

2) The benchmark scheme in [31] is termed as maxEEOPSNR, which aims for minimizing the power consumption via finding the optimal SINR and blocklength.

3) The proposed schemes in [37] is named as maxEE-PBO, where the arrived power of MTC devices in the same subchannel are gradually degraded with a step of $\varrho \mathrm{dB}$.

4) To evaluate the tradeoff between the achievable effective $\mathrm{EE}$ and rate, we compare the algorithm of maximizing achievable effective rate in NOMA system, which is termed as maxRate-NOMA.

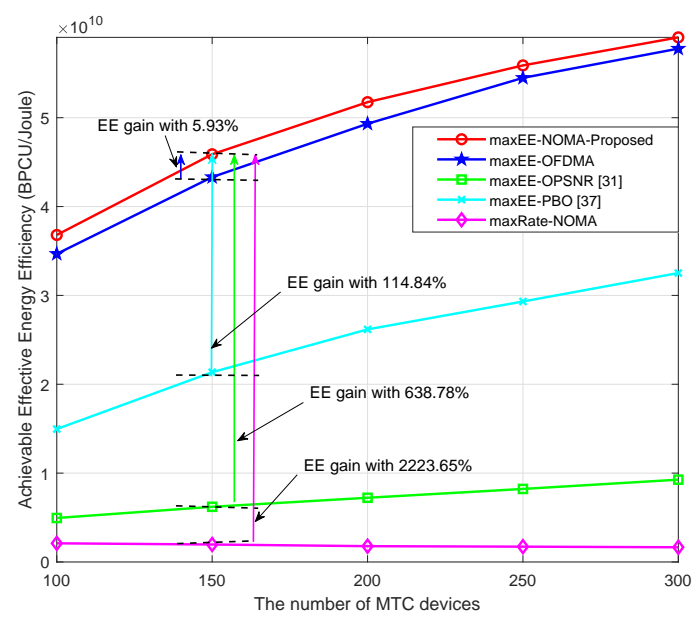

Fig. 3. Available effective EE versus number of MTC devices

In Fig. 3, we evaluated the performance of achievable effective EE for the proposed maxEE-NOMA and other benchmark algorithms. The total number of MTC devices ranges from 100 to 300 and the upper limit number of MTC devices per subchannel is $N_{n}=2$. The blocklength is 392 symbols and the decoding error probability is $10^{-5}$. The achievable effective EE of the maxEE-NOMA-proposed outperforms other four algorithms. Especially, the proposed maxEE-NOMA in achievable effective EE is more than 2000 times that of the benchmark maxRate-NOMA scheme. This is because the MTC devices strive to maximize the achievable effective rate with large transmission power in maxRate-NOMA. While the MTC devices will try their best to reduce the transmission power to maximize the achievable effective EE in maxEENOMA. Hence, The transmission power in maximizing their achievable effective rate is larger than that in maximizing their achievable effective EE. It means that performing proper power allocation can make the energy of MTC devices more efficient. Furthermore, the channel gain of MTC devices in the proposed maxEE-NOMA scheme are better. They can deliver more bits with lower transmission power and obtain a good performance in terms of achievable effective EE. Compared with maxEE-OFDMA, maxEE-PBO [37], maxEEOPSNR [31], the proposed maxEE-NOMA are more suitable for energy-efficient short packet communications in massive MTC networks.

The achievable effective rate of different schemes are evaluated in Fig. 4, where the simulation conditions are the same as in Fig. 3. Compared with maxRate-NOMA, the algorithms maximizing achievable effective EE have a rate loss. As seen in Fig. 3 and Fig. 4 the maxEE-NOMA-proposed obtains huge gain in achievable effective $\mathrm{EE}$ at the cost of $50.15 \%$ loss in the achievable effective rate. It is caused by the tradeoff between achievable effective EE and rate in short packet communication. Because the maxEE-OPSNR minimize the power consumption of MTC devices without considering the circuit power consumption, the performance of it in 


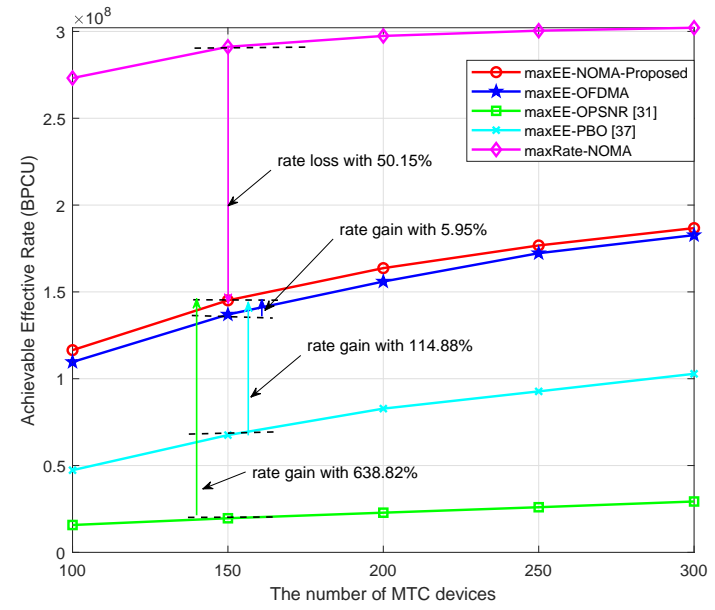

Fig. 4. Available effective rate versus the number of MTC devices

achievable effective rate and EE are poor. For the energysensitive MTC devices, it is better to adjust their power to maximize the achievable effective EE, because it will transmit more information data bits with less Joule consumed.

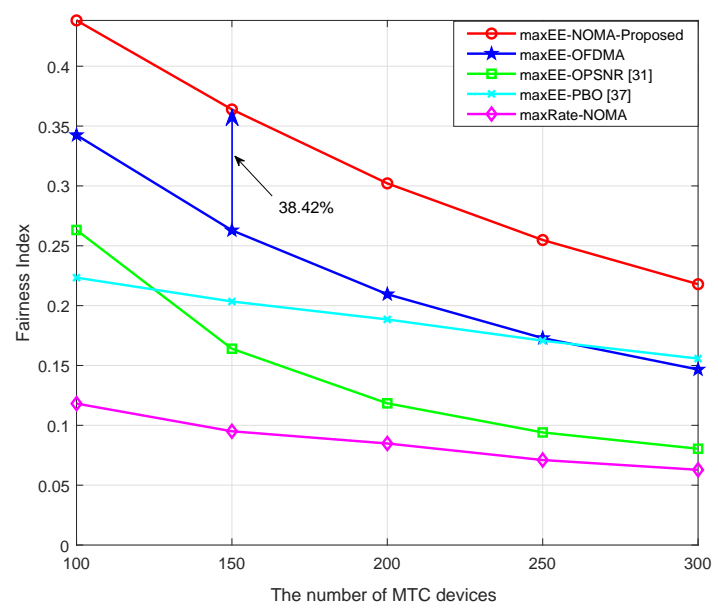

Fig. 5. Jain fairness index versus number of MTC devices

Because the fairness is an important metric to measure the limited subchannel and power allocation, we introduce the Jain's fairness index $[46]$ as Fairness index $=\frac{\left(\sum_{m=1}^{M} E E_{m}\right)^{2}}{M \sum_{m=1}^{M}\left(E E_{m}\right)^{2}}$. With the same simulation conditions in Fig. 3 and Fig. 4 . the fairness performance versus the number of MTC devices is shown in Fig. 5 Since the difference among maxRateNOMA, maxEE-OPSNR and maxEE-NOMA-proposed is the transmission power, their corresponding fairness curves follow the same trend. Affected by the introduced interference in NOMA, the power allocation is the main factor when the number of MTC devices increases. Therefore, their Jain Fairness Index decreases as the number of MTC devices competing for the limited resources increases. Compared with the maxEE-OFDMA $\left(N_{n}=1\right)$ scheme, although the maxEENOMA-proposed $\left(N_{n}=2\right)$ scheme obtains gains in available effective EE and rate are $5.93 \%$ and $5.95 \%$, the gain in fairness is $38.42 \%$. Furthermore, constrained by the back-off power control, the transmission power and introduced interference are limited, thus the Jain fairness index of maxEE-PBO is decreasing slowly. From Fig. 5, we obtain that the maxEENOMA-proposed also has a better performance in fairness.

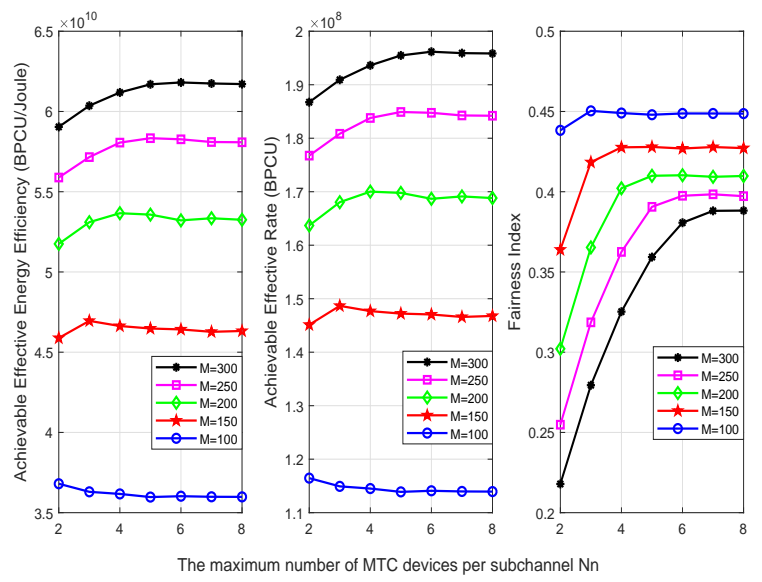

Fig. 6. System performances with the different maximum number of MTC devices $N_{n}$ per subchannel

The Fig. 6 presents the performance of available effective $\mathrm{EE}$, rate and fairness versus the maximum number of MTC devices $N_{n}$ per subchannel. The total number of subchannels is $N=50$. For fixed the total number of MTC devices $M$, there is a critical value of $N_{n}$ for the system performance. The available effective EE increases with $N_{n}$ when $N_{n} \times N<M$ and is up to the maximum when $N_{n} \times N=M$. However, the loss generated by the multi-user interference is over the gain brought by the subchannel sharing. Compared with the maximum, the available effective EE has a slight decrease when $N_{n} \times N>M$. The performance of the available effective rate follows a similar trend of that of available effective EE. While in the performance of fairness, the fairness index increases with $N_{n}$ when $N_{n}$ is smaller than the critical value. Then the fairness performance may not be improved by increasing $N_{n}$. Furthermore, the critical value increases with the total number of MTC devices, because the MTC devices have more chances to select their preference sub-channels.

The performance of available effective EE versus blocklength $L_{m}$ is evaluated in Fig. 7 The blocklength ranges from 392 1832 symbols. The achievable effective rate is a monotonically increasing function of blocklength [24]. When the blocklength increases to a certain value, the gain of rate approximates to zero. Thus, the achievable effective EE in Fig. 7 first increases with the blocklength $L_{m}$, then converges to a certain value. It means that a suitable blocklength will benefit for the energy efficient short packet transmission. Although the influence of blocklength on achievable effective EE is negligible with fixed number of MTC devices, it becomes more and more important when the number of MTC devices increases. Therefore, in future we will further study how to choose a suitable blocklength to maximize the achievable effective EE of each MTC device. 


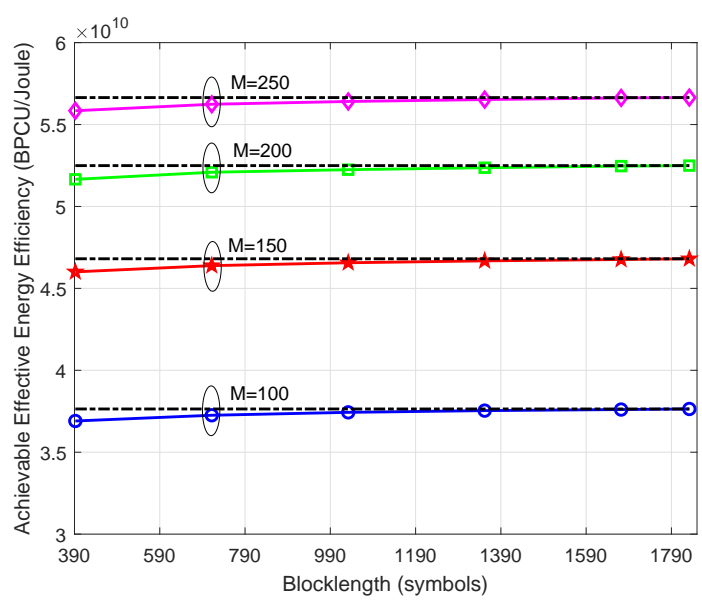

Fig. 7. Available effective EE versus blocklength $L_{m}$

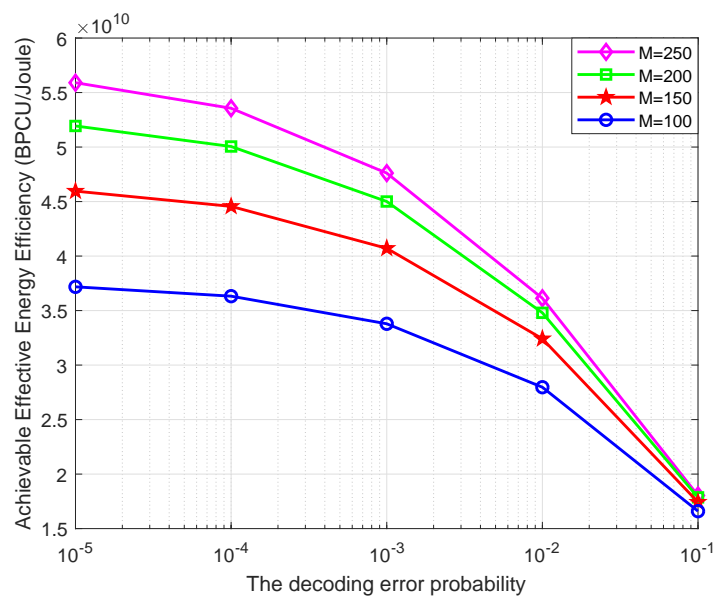

Fig. 8. Available effective EE versus the decoding error probability $\varepsilon$

The performance of achievable effective EE versus the decoding error probability is evaluated in Fig. 8 . Due to the decoding error probability, the SIC technology cannot be perfectly performed in removing the multiuser co-channel interference. Meanwhile, the larger the probability of decoding errors, the more residual co-channel interference will be left to degrade the performance. Another reason is we consider the achievable effective EE, large decoding error probability will also reduce the successful probability of receiving data. The reliability and the EE are a pair of contradictories to measure the system's performance. Thus, the achievable effective EE decreases dramatically with the decoding error probability $\varepsilon$ increasing.

The achievable effective EE with respect to the maximum transmission power $P_{\max }$ of each MTCD is demonstrated in Fig. 9 The maximum transmission power of MTC devices varies from $0 \mathrm{dBm}$ to $30 \mathrm{dBm}$. With a fixed number of MTC devices, since the increased achievable effective rate is greater than the increased power, the achievable effective EE increases with the maximum transmission power if $P_{\max } \leq 12 \mathrm{dBm}$. However, the achievable effective EE has a slight decrease when $P_{\max }>12 \mathrm{dBm}$. This is because large power will generate more penalty to the achievable effective rate. Furthermore, multiuser interference in the same channel will be greatly aggravated due to the increased transmission power. Therefore, the achieved sum rate of all MTC devices grows slower than the total transmission power consumption. For better performance in achievable effective EE, the MTC devices will choose lower transmission power and $P_{m, n} \leq 12$ $\mathrm{dBm}$.

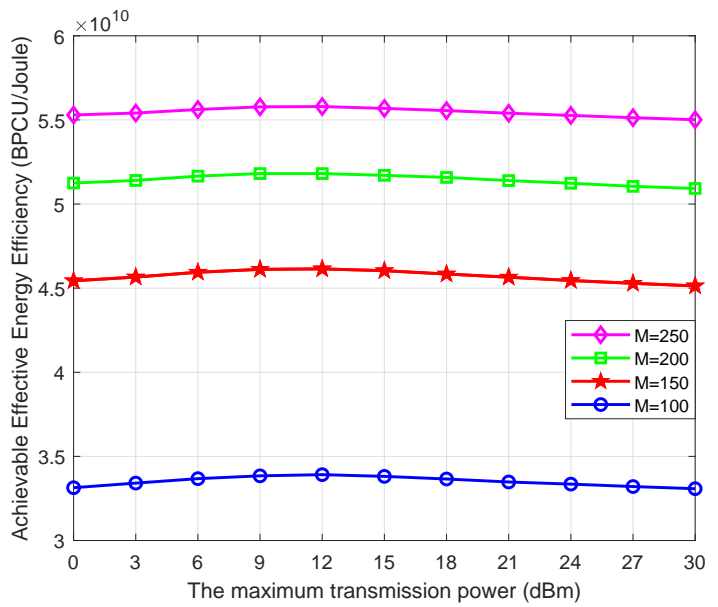

Fig. 9. Available effective EE versus the maximum transmission power $P_{\max }$

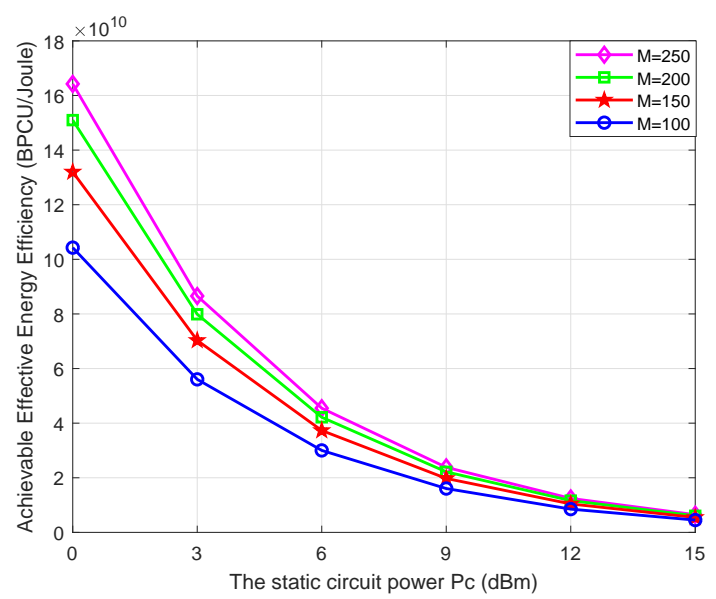

Fig. 10. Available effective EE versus the static circuit power $P_{m, C}$

The performance of achievable effective EE versus the static circuit power $P_{m, C}$ of each MTCD is shown in Fig. 10. The static circuit power of MTC devices varies from $0 \mathrm{dBm}$ to $15 \mathrm{dBm}$. Fixed the number of MTC devices and maximum transmission power, the achievable effective EE decreases with the static circuit power. Due to the hardware limitation of MTC devices, with the increase of static circuit power, the influence of the number of MTC devices on achievable effective EE becomes smaller and smaller, and static circuit power becomes the main factor affecting SEE. Thus, it is important to design the MTC devices with high energy and cost efficiencies or develop more energy-efficient technology to compensate the energy consumption in the static circuit. For 
example, the energy harvesting technology can be exploited for MTC devices with long lifetime requirements.

\section{CONCLUSION}

Different with HTC, massive MTC has different characteristics in terms of uplink dominant, short packet communications and energy efficient. This paper maximizes the achievable effective EE of massive MTC devices in uplink NOMA-based short packet communications. The decoding error probability in receiver and the minimum data rate requirements of MTC devices are taken into consideration. Moreover, we set an upper bound for the number of MTC devices sharing the same subchannels to reduce the decoding error probability and complexity of receiver. The formulated optimization problem is decoupled into subchannel allocation problem and power allocation problem. Furthermore, we formulate an efficient Q-learning algorithm to allocate the subchannels and approximated the achievable effective rate in uplink NOMAbased short packet communications to obtain the optimal transmission power. Simulations validate that the proposed scheme can significantly improve the achievable effective EE of MTC devices. In addition, we mainly study the influence of finite blocklength packets and decoding error probability in this paper, while the data arrival rate, delay and the secure transmission are not involved. In future, we will further investigate the optimal resource allocation in short packet communications for uplink NOMA-based massive MTC networks by considering the influences of aforementioned factors.

\section{APPENDIX A}

\section{PROOF OF LEMMA 1}

Proof: Based on 10 , we have $R=f(\rho)$, then

1) Positive $R \geq 0$

$$
f(\rho)=\log _{2}(1+\rho)-\kappa \frac{\sqrt{\rho(\rho+2)}}{1+\rho} \geq 0
$$

Thus, we can obtain

$$
\kappa \leq \frac{\log _{2}(1+\rho)(1+\rho)}{\sqrt{\rho(\rho+2)}}=g(\rho)
$$

2) Monotonically increasing $\frac{\partial R}{\partial \rho} \geq 0$

$$
\begin{gathered}
f^{\prime}(\rho)=\frac{1}{\ln 2(1+\rho)}-\frac{\kappa}{(1+\rho)^{2} \sqrt{\rho(\rho+2)}} \geq 0 \\
\kappa \leq \frac{(1+\rho) \sqrt{\rho(\rho+2)}}{\ln 2}=g_{1}(\rho)
\end{gathered}
$$

It is obviously that $g_{1}(\rho) \geq g(\rho)$

3) Concave $\frac{\partial^{2} R}{\partial \rho^{2}} \leq 0$

$$
\begin{aligned}
f^{\prime \prime}(p) & =-\frac{1}{\ln 2(1+\rho)^{2}}+\frac{\kappa}{(1+\rho)^{3}[\rho(\rho+2)]^{\frac{3}{2}}} \\
& +\frac{3 \kappa}{(1+\rho)^{3} \sqrt{\rho(\rho+2)}} \\
& =\frac{\left[\ln 2 \kappa\left(3 \rho^{2}+6 \rho+1\right)-(1+\rho)[\rho(\rho+2)]^{\frac{3}{2}}\right]}{\ln 2(1+\rho)^{3}[\rho(\rho+2)]^{\frac{3}{2}}}
\end{aligned}
$$

If $f^{\prime \prime}(\rho) \leq 0$, then we have $\ln 2 \kappa\left(3 \rho^{2}+6 \rho+1\right)-$ $(1+\rho)[\rho(\rho+2)]^{\frac{3}{2}} \leq 0$, and

$$
\kappa \leq \frac{(1+\rho)[\rho(\rho+2)]^{\frac{3}{2}}}{\ln 2\left(3 \rho^{2}+6 \rho+1\right)}=g_{2}(p)
$$

$g(\rho), g_{2}(\rho)$ are monotonically increasing functions

$$
\left\{\begin{array}{lc}
g_{2}(\rho) \leq g(\rho) & 0 \leq \rho \leq \rho_{0} \\
g_{2}(\rho)>g(\rho) & \rho>\rho_{0}
\end{array}\right.
$$

where $\rho_{0}$ is the positive solution of equation $g_{2}\left(\rho_{0}\right)=g\left(\rho_{0}\right)$. Furthermore, we define $\vartheta=g_{2}\left(\rho_{0}\right)=g\left(\rho_{0}\right)$.

- $\kappa>\vartheta$, i.e., $\kappa>\vartheta=g_{2}\left(\rho_{0}\right)=g\left(\rho_{0}\right)$, this mean that $\rho_{0} \leq g_{2}^{-1}(\kappa) \leq g^{-1}(\kappa)$. To guarantee the non-negative finite block-length achievable rate, $\rho \geq g^{-1}(\kappa)$. Therefore, this condition implies that $\rho \geq g^{-1}(\kappa) \geq g_{2}^{-1}(\kappa)$, which satisfies $\kappa \leq g_{2}(\rho)$ that $R$ is concave.

- $\kappa \leq \vartheta$, i.e., $\kappa \leq \vartheta=g_{2}\left(\rho_{0}\right)=g\left(\rho_{0}\right)$, this mean that $\rho_{0} \geq g_{2}^{-1}(\kappa) \geq g^{-1}(\kappa)$. To guarantee the nonnegative finite block-length achievable rate, $\rho \geq g^{-1}(\kappa)$. Therefore, there are two possible cases: 1) $\rho \geq g_{2}^{-1}(\kappa) \geq$ $g^{-1}(\kappa)$, then $\kappa \leq g_{2}(\rho)$ and $R$ is concave. 2) $g_{2}^{-1}(\kappa) \geq$ $\rho \geq g^{-1}(\kappa)$, then $\kappa \geq g_{2}(\rho)$ and $\frac{\partial^{2} R}{\partial \rho^{2}} \geq 0$, hence $R$ is convex.

\section{APPENDIX B}

PROOF OF THEOREM 1

Proof: According to (19), we have $U_{R}(\mathbf{P})=$ $\sum_{n=1}^{N} \sum_{m=1}^{M_{n}} \bar{R}_{m, n}$ and $U_{T P}(\mathbf{p})=P_{\text {total }}$. Therefore, we have

$$
\eta^{*}=\frac{U_{R}\left(\mathbf{p}^{*}\right)}{U_{T P}\left(\mathbf{p}^{*}\right)} \geq \frac{U_{S C}(\mathbf{p})}{U_{T P}(\mathbf{p})}
$$

and

$$
\begin{aligned}
& U_{R}(\mathbf{p})-\eta^{*} U_{T P}(\mathbf{p}) \leq 0 \\
& U_{R}\left(\mathbf{p}^{*}\right)-\eta^{*} U_{T P}\left(\mathbf{p}^{*}\right)=0
\end{aligned}
$$

We can obtain that $\max _{\left\{p_{m, n}^{*}\right\}}\left\{U_{S C}(\mathbf{p})-\eta^{*} U_{T P}(\mathbf{p})\right\}=0$. Thus, the sufficient condition of Theorem 1 has been proved.

Then, the necessary condition should be proved. If $\left\{\mathbf{p}^{*}\right\}$ is the optimal power allocation policy, we have $U_{R}\left(\mathbf{p}^{*}\right)-$ $\eta^{*} U_{T P}\left(\mathbf{p}^{*}\right)=0$. Then, for any feasible $\{\mathbf{p}\}$, we can obtain

$$
\begin{aligned}
U_{R}(\mathbf{p}) & -\eta^{*} U_{T P}(\mathbf{p}) \\
& \leq U_{R}\left(\mathbf{p}^{*}\right)-\eta^{*} U_{T P}\left(\mathbf{p}^{*}\right)=0
\end{aligned}
$$

Hence,

$$
\frac{U_{R}\left(\mathbf{p}^{*}\right)}{U_{T P}\left(\mathbf{p}^{*}\right)}=\eta^{*}, \frac{U_{R}(\mathbf{p})}{U_{T P}(\mathbf{p})} \leq \eta^{*}
$$

Therefore, the optimal power allocation strategies $\mathbf{p}^{*}$ of the transformed objective function is also the optimal ones of the original objective function. 


\section{REFERENCES}

[1] Cisco, "Cisco visual networking index: Global mobile data traffic forecast update, 2017-2022," Tech. Rep., Feb 2019. [Online]. Available: https://www.cisco.com/c/en/us/solutions/collateral/service-provider/ visual-networking-index-vni/white-paper-c11-738429.pdf

[2] C. Bockelmann, N. Pratas, H. Nikopour, K. Au, T. Svensson, C. Stefanovic, P. Popovski, and A. Dekorsy, "Massive machine-type communications in 5G: physical and MAC-layer solutions," IEEE Commun. Mag., vol. 54, no. 9, pp. 59-65, Sep. 2016.

[3] F. Boccardi, R. W. Heath, A. Lozano, T. L. Marzetta, and P. Popovski, "Five disruptive technology directions for 5G," IEEE Commun. Mag., vol. 52, no. 2, pp. 74-80, Feb. 2014.

[4] Z. Ma, M. Xiao, Y. Xiao, Z. Pang, H. V. Poor, and B. Vucetic, "High-reliability and low-latency wireless communication for Internet of Things: Challenges, fundamentals and enabling technologies," IEEE Internet Things J., pp. 1-25, 2019.

[5] Z. Dawy, W. Saad, A. Ghosh, J. G. Andrews, and E. Yaacoub, "Toward massive machine type cellular communications," IEEE Wireless Commun., vol. 24, no. 1, pp. 120-128, Feb. 2017.

[6] S. Krishna Sharma and X. Wang, "Towards massive machine type communications in ultra-dense cellular IoT networks: Current issues and machine learning-assisted solutions," arXiv:1808.02924, 2018. [Online]. Available: http://arxiv.org/abs/1808.02924

[7] Z. Ding, X. Lei, G. K. Karagiannidis, R. Schober, J. Yuan, and V. K. Bhargava, "A survey on non-orthogonal multiple access for 5G networks: Research challenges and future trends," IEEE J. Sel. Areas Commun. vol. 35 , no. 10, pp. 2181-2195, Oct. 2017.

[8] G. Durisi, T. Koch, and P. Popovski, "Toward massive, ultrareliable, and low-latency wireless communication with short packets," Proc. IEEE, vol. 104, no. 9, pp. 1711-1726, Sep. 2016

[9] Y. Polyanskiy, H. V. Poor, and S. Verdu, "Channel coding rate in the finite blocklength regime," IEEE Trans. on Inf. Theory, vol. 56, no. 5, pp. 2307-2359, May 2010.

[10] E. Dosti, U. L. Wijewardhana, H. Alves, and M. Latva-aho, "Ultra reliable communication via optimum power allocation for type-I ARQ in finite block-length," in 2017 IEEE International Conference on Communications (ICC). Paris, France: IEEE, May 2017, pp. 1-6.

[11] Y. Li, W. Xiong, N. Sullivan, G. Chen, B. Chen, G. HadYnski, C. Banner, Y. Xu, and X. Tian, "Power optimization for energy efficient wireless communications using Hybrid-ARQ," in MILCOM 2018 - 2018 IEEE Military Communications Conference (MILCOM), Los Angeles, CA, USA, Oct 2018, pp. 1062-1067.

[12] Y. Hu, M. Serror, K. Wehrle, and J. Gross, "Finite blocklength performance of cooperative multi-terminal wireless industrial networks," IEEE Trans. Veh. Technol., vol. 67, no. 7, pp. 5778-5792, Jul. 2018.

[13] L. Zhang and Y. Liang, "Average throughput analysis and optimization in cooperative IoT networks with short packet communication," IEEE Trans. Veh. Technol., vol. 67, no. 12, pp. 11 549-11 562, Dec. 2018.

[14] B. Makki, C. Fang, T. Svensson, M. Nasiri-Kenari, and M. Zorzi, "Delay-sensitive area spectral efficiency: A performance metric for delay-constrained green networks," IEEE Trans. Wireless Commun., vol. 65 , no. 6, pp. 2467-2480, June 2017.

[15] C. She, C. Yang, and T. Q. S. Quek, "Radio resource management for ultra-reliable and low-latency communications," IEEE Commun. Mag., vol. 55, no. 6, pp. 72-78, June 2017.

[16] J. Chen, X. Ge, and Q. Ni, "Coverage and handoff analysis of $5 \mathrm{G}$ fractal small cell networks," IEEE Trans. Wireless Commun., vol. 18, no. 2, pp. 1263-1276, Feb 2019.

[17] X. Ge, J. Yang, H. Gharavi, and Y. Sun, "Energy efficiency challenges of 5G small cell networks," IEEE Commun. Mag., vol. 55, no. 5, pp. 184-191, May 2017.

[18] X. Ge, Y. Sun, H. Gharavi, and J. Thompson, "Joint optimization of computation and communication power in multi-user massive MIMO systems," IEEE Trans. Wireless Commun., vol. 17, no. 6, pp. 40514063, June 2018.

[19] W. Hao, Z. Chu, F. Zhou, S. Yang, G. Sun, and K. Wong, "Green communication for NOMA-based CRAN," IEEE Internet Things J., vol. 6, no. 1, pp. 666-678, Feb 2019

[20] S. Han, X. Xu, S. Fang, Y. Sun, Y. Cao, X. Tao, and P. Zhang, "Energy efficient secure computation offloading in NOMA-based mMTC networks for IoT,' IEEE Internet Things J., vol. 6, no. 3, pp. 5674-5690, June 2019.

[21] B. Makki, T. Svensson, and M. Zorzi, "Wireless energy and information transmission using feedback: Infinite and finite block-length analysis," IEEE Trans. Wireless Commun., vol. 64, no. 12, pp. 5304-5318, Dec. 2016.
[22] Y. Hu, Y. Zhu, M. C. Gursoy, and A. Schmeink, "Swipt-enabled relaying in iot networks operating with finite blocklength codes," IEEE J. Sel. Areas Commun., vol. 37, no. 1, pp. 74-88, Jan. 2019.

[23] J. Chen, L. Zhang, Y. Liang, X. Kang, and R. Zhang, "Resource allocation for wireless-powered IoT networks with short packet communication," IEEE Trans. Wireless Commun., vol. 18, no. 2, pp. 1447-1461, Feb. 2019.

[24] S. Xu, T. Chang, S. Lin, C. Shen, and G. Zhu, "Energy-efficient packet scheduling with finite blocklength codes: Convexity analysis and efficient algorithms," IEEE Trans. Wireless Commun., vol. 15, no. 8, pp. 5527-5540, Aug. 2016.

[25] A. Avranas, M. Kountouris, and P. Ciblat, "Energy-latency tradeoff in ultra-reliable low-latency communication with retransmissions," IEEE J. Sel. Areas Commun., vol. 36, no. 11, pp. 2475-2485, Nov. 2018.

[26] C. Sun, C. She, C. Yang, T. Q. S. Quek, Y. Li, and B. Vucetic, "Optimizing resource allocation in the short blocklength regime for ultra-reliable and low-latency communications," IEEE Trans. Wireless Commun., vol. 18, no. 1, pp. 402-415, Jan. 2019.

[27] S. Han, X. Xu, X. Tao, and P. Zhang, "Joint power and sub-channel allocation for secure transmission in NOMA-based mMTC networks," IEEE Syst. J., vol. 13, no. 3, pp. 2476-2487, Sep. 2019.

[28] X. Lai, Q. Zhang, and J. Qin, "Cooperative NOMA short-packet communications in flat rayleigh fading channels," IEEE Trans. Veh. Technol., pp. 1-5, 2019.

[29] Z. Ding, L. Dai, and H. V. Poor, "MIMO-NOMA design for small packet transmission in the internet of things," IEEE Access, vol. 4, pp. 13931405, 2016.

[30] X. Sun, S. Yan, N. Yang, Z. Ding, C. Shen, and Z. Zhong, "Shortpacket downlink transmission with non-orthogonal multiple access," IEEE Trans. Wireless Commun., vol. 17, no. 7, pp. 4550-4564, Jul. 2018.

[31] Y. Xu, C. Shen, T.-H. Chang, S.-C. Lin, Y. Zhao, and G. Zhu, "On energy-efficient NOMA designs for heterogeneous low-latency downlink transmissions," arXiv:1806.09144, 2018. [Online]. Available: http://arxiv.org/abs/1806.09144

[32] R. Abbas, M. Shirvanimoghaddam, Y. Li, and B. Vucetic, "A multi-layer grant-free NOMA scheme for short packet transmissions," in 2018 IEEE Global Communications Conference (GLOBECOM), Abu Dhabi, United Arab Emirates, Dec. 2018, pp. 1-6.

[33] J. J. Dinkel, G. A. Kochenberger, and W. H. Elliott, "A linear programming approach to geometric programs," Naval Research Logistics Quarterly, vol. 25, no. 1, pp. 39-53, 1978.

[34] D. Qiao, M. C. Gursoy, and S. Velipasalar, "Transmission strategies in multiple-access fading channels with statistical QoS constraints," IEEE Trans. Inf. Theory, vol. 58, no. 3, pp. 1578-1593, March 2012.

[35] Y. Gao, B. Xia, Y. Liu, Y. Yao, K. Xiao, and G. Lu, "Analysis of the dynamic ordered decoding for uplink NOMA systems with imperfect CSI," IEEE Trans. Veh. Technol., vol. 67, no. 7, pp. 6647-6651, July 2018.

[36] N. Mokari, M. Moltafet, and A. Zakeri, "Joint radio resource allocation and sic ordering in NOMA-based networks using submodularity and matching theory," IEEE Trans. Veh. Technol., pp. 1-1, 2019.

[37] N. Zhang, J. Wang, G. Kang, and Y. Liu, "Uplink nonorthogonal multiple access in 5G systems," IEEE Commun. Lett., vol. 20, no. 3, pp. 458-461, Mar. 2016.

[38] X. Chen, R. Jia, and D. W. K. Ng, "On the design of massive non-orthogonal multiple access with imperfect successive interference cancellation," IEEE Trans. on Commun., vol. 67, no. 3, pp. 2539-2551, Mar. 2019.

[39] X. Chen, Z. Zhang, C. Zhong, R. Jia, and D. W. K. Ng, "Fully non-orthogonal communication for massive access," IEEE Trans. on Commun., vol. 66, no. 4, pp. 1717-1731, Apr. 2018.

[40] R. S. Sutton and A. G. Barto, Reinforcement learning: An introduction. MIT press Cambridge, 1998.

[41] S. Boyd and L. Vandenberghe, Convex optimization. Cambridge university press, 2004.

[42] J. Papandriopoulos and J. S. Evans, "Scale: A low-complexity distributed protocol for spectrum balancing in multiuser DSL networks," IEEE Trans. on Inf. Theory, vol. 55, no. 8, pp. 3711-3724, Aug. 2009.

[43] 3GPP, "3rd generation partnership project; technical specification group radio access network; evolved universal terrestrial radio access (EUTRA); further advancements for E-UTRA physical layer aspects," TR 36.814, Release 9, Mar. 2017.

[44] _ _ "3rd generation partnership project; technical specification group radio access network; study on non-orthogonal multiple access (NOMA) for NR," TR 38.812, Release 16, Dec. 2018. 
[45] P. Popovski, K. F. Trillingsgaard, O. Simeone, and G. Durisi, "5G wireless network slicing for eMBB, URLLC, and mMTC: A communicationtheoretic view," IEEE Access, vol. 6, pp. 55 765-55 779, 2018.

[46] R. K. Jain, D.-M. W. Chiu, and W. R. Hawe, "A quantitative measure of fairness and discrimination," Eastern Research Laboratory, Digital Equipment Corporation, Hudson, MA, Sep. 1984.

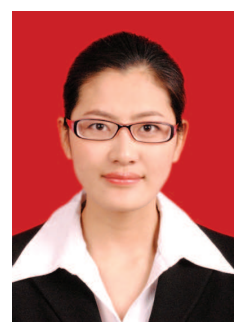

Shujun Han (S'18) is a Ph.D. in the School of Information and Communication Engineering, Beijing University of Posts and Telecommunications, Beijing, China. She received the B.E. and M.S. degrees from Zhengzhou University in 2013 and 2016. Her main research interests are in the area in cognitive radio networks, machine type communications and networks, Internet of Things, with currently focusing on radio resource management for enhancing the energy efficient physical layer security.

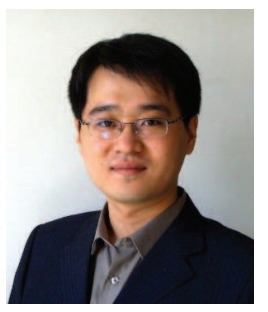

Xiaodong Xu (S'06-M'07-SM'18) received his B.S degree in Information and Communication Engineering and Master's Degree in Communication and Information System both from Shandong University in 2001 and 2004 separately. He received his Ph.D. degrees of Circuit and System in Beijing University of Posts and Telecommunications (BUPT) in 2007. $\mathrm{He}$ is currently a professor of BUPT. He has coauthored seven books and more than 120 journal and conference papers. He is also the inventor or coinventor of 37 granted patents. His research interests cover network architecture, moving network, coordinated multi-point, mobile network visualization and IoT. He is supported by Beijing Nova Programme on mobile networking.

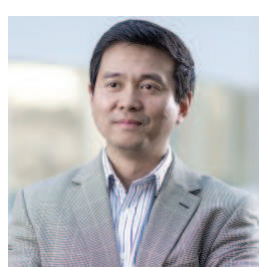

Pei Xiao is a Professor in Wireless Communications at ICS, home of 5GIC. He received the B. Eng, $\mathrm{MSc}$ and $\mathrm{PhD}$ degree from Huazhong University of Science and Technology, Tampere University of Technology, Chalmers University of Technology, respectively. Prior to joining Surrey in 2011, he worked at Newcastle University and Queen's University Belfast and had held positions at Nokia Network in Finland. He is the technical manager of 5GIC, leading and coordinating research activities and overseeing major projects in 5GIC. His main research interests and expertise span a wide range of areas in communications theory and signal processing for wireless communications.

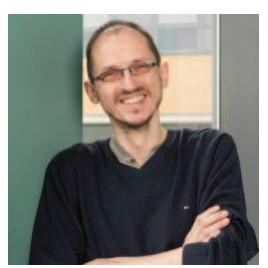

Klaus Moessner is a Professor of cognitive networks with the 5G Innovation Centre (5GIC), Institute for Communication Systems, University of Surrey, U.K. He has been actively involved in the various European Community funded research frameworks (from FP 5 onward), was involved in some $20 \mathrm{EU}$ funded projects, has been a Technical Manager and a Project Manager, and is currently leading the FP7 Project SocIoTal and the H2020 EUJapan Project iKaaS. He was the Founding Chair of the IEEE DySPAN Working Group 6 on Spectrum Sensing Interfaces. His research interests are around the aspects of reconfigurability on the different system levels, including reconfiguration management and scheduling in wireless networks as well as network supported adaptability of multimodal user interfaces.

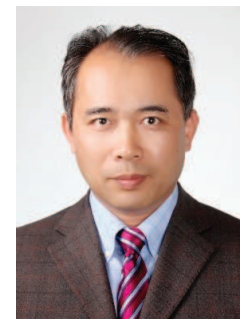

Xiaofeng Tao (S'99-A'02-M'03-SM'13) received the B.S. degree in electrical engineering from Xian Jiaotong University, Xi'an, China, in 1993, and the M.S.E.E. and Ph.D. degrees in telecommunication engineering from the Beijing University of Posts and Telecommunications (BUPT), Beijing, China, in 1999 and 2002, respectively. He was a Visiting Professor with Stanford University, Stanford, CA USA, from 2010 to 2011; the Chief Architect with the Chinese National FUTURE Fourth-Generation (4G) TDD Working Group from 2003 to 2006; and established the 4G TDD CoMP Trial Network in 2006. He is currently a Professor with the BUPT and a fellow of the Institution of Engineering and Technology. He is the inventor or co-inventor of 50 patents and the author or co-author of 120 papers in $4 \mathrm{G}$ and beyond $4 \mathrm{G}$. He is currently involved in fifth-generation networking technology and mobile network security.

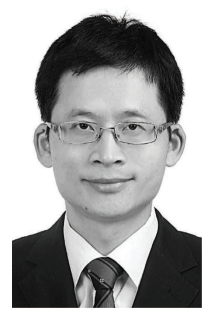

Zilong Liu is a Senior Research Fellow in the 5G Innovation Centre, University of Surrey. He received his $\mathrm{PhD}$ in 2014 from the School of Electrical and Electronics Engineering, Nanyang Technological University (NTU), Singapore. He received his M.S. degree in the Department of Electronic Engineering from Tsinghua University and B.S. degree in the School of Electronics and Information Engineering from Huazhong University of Science and Technology (HUST), in 2007 and 2004, respectively. He has been awarded a two-year (2018-2019) Research Fellowship for International Young Scientists funded by National Natural Science Research Foundation of China. Supported by the government of India, Dr Liu was a Global Initiatives of Academic Network (GIAN) Course Instructor in 2018 at Indian Institutes of Technology (IIT) Patna. Dr Liu is an Associate Editor of IEEE Access since January 2017. He is generally interested in coding and signal processing for various communication systems with emphasis on waveform design, algebraic coding, error correction codes, iterative receiver design, as well as robust/efficient multiuser communications. His current research focus is the physical layer of $5 \mathrm{G}$ and beyond.

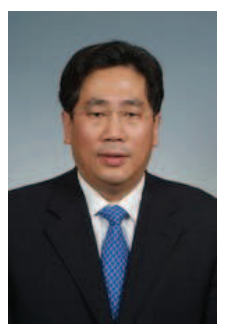

Ping Zhang (M'07-SM'15-F'18) received his Ph.D. degree from BUPT in 1990. He is currently a professor at Beijing University of Posts and Telecommunications. He has published eight books and more than 400 papers, and he holds approximately 170 patents. He is Executive Associate Editor-in-chief on information sciences of Chinese Science Bulletin, a Member of next-generation broadband wireless communication network in National Science and Technology Major Project committee, a Member of the 5th Advisory Committee of NSFC(National Natural Science Foundation of China), the Chief Scientist of "973" National Basic Research Program of China, member of MOST(The Ministry of science and technology) 863 Program Expert team, member of MOST IMTAdvanced 5G Expert team. His current research interests include mobile communications, ubiquitous networking, and service provisioning, especially in the key techniques of the $5 \mathrm{G}$ systems. 